\title{
Ventricular Tachycardia Radiofrequency Ablation with Extracorporeal Membrane Oxygenation
}

\author{
Avi Sabbag, Roy Beinart, Michael Eldar, Osnat Gurevitz, Amihay Shinfeld, Jacob Lavee, Ehud \\ Raanani, Alexander Kogan, Dan Spiegelstein, Michael Glikson and Eyal Nof*
}

Davidai Arrhythmia Service and Cardiac Surgery Department, Leviev Heart Center, Sheba Medical Center Ramat Gan, Israel

*Corresponding author: Eyal Nof, Davidai Arrhythmia Service and Cardiac Surgery Department, Leviev Heart Center, Chaim Sheba Medical Center, Tel-Hashomer 52621, Israel, Tel: +972302608; E-mail: Eyal.Nof@sheba.health.gov.il

Rec Date: Oct 19, 2016, Acc Date: Dec 18, 2016, Pub Date: Dec 20, 2016

Citation: Sabbag A, Beinart R, Eldar M, et al. Ventricular Tachycardia Radiofrequency Ablation with Extracorporeal Membrane Oxygenation. Interv Cardiol J 2016, 2:3.

\section{Abstract}

Introduction: Cases of ventricular tachycardia (TV) with hemodynamic compromise present a challenge in achieving non-inducibility by radiofrequency catheter ablation (RFCA). We report our experience of VT RFCA facilitated by elective mechanical circulatory support.

Methods and results: Five patients with hemodynamically unstable, recurrent ventricular arrhythmias that were unresponsive to medical therapy underwent extracorporeal membrane oxygenation (ECMO) assisted RFCA of scar related VT. All underwent RFCA under general anesthesia and were connected to an ECMO circuit maintained at minimum flow of $1.5 \mathrm{~L} / \mathrm{min}$. In case of VT or VF the blood flow of the ECMO circuit was increased to $4 \mathrm{~L} / \mathrm{min}$ to allow hemodynamic stability and adequate systemic organ perfusion. A total of 8 VTs were observed. In 4 cases, we mapped during VT the critical isthmus was found and ablated. Four VTs were targeted by substrate mapping only. Complete success, defined as non-inducibility with aggressive program stimulation of any VT, was achieved in 4 patients. In a single patient, a non-clinical VT was still inducible. He died of septic shock 24 hour after the procedure. The remaining 4 were free of ventricular arrhythmia as proven by implanted defibrillator interrogation, over a median follow up of 16 months.

Conclusion: ECMO implantation for VTRFCA is safe and assists in reaching the desired endpoint of noninducibility. This approach should be considered in high risk patients who may not otherwise tolerate such procedures.

Keywords: Ventricular tachycardia, Extracorporeal membrane oxygenation, Radiofrequency ablation, Noninducible

\section{Introduction}

Radiofrequency catheter ablation (RFCA) has an established role in the treatment of drug refractory ventricular tachycardia (VT) [1]. However, patients with recurrent VTs uncontrolled by medications, in which the clinical VT is accompanied with hemodynamic compromise, comprise a very high risk population with limited therapeutic options. Whether performing ablation during VT has an advantage over substrate approach ablation only is still unresolved $[2,3]$. RFCA during VT has the advantage of allowing better identification of critical VT isthmuses and may lead to more precise ablation. Even procedures limited to substrate modification may result in a significant risk of acute hemodynamic decompensation and increased risk of procedure related mortality. In several studies, non inducibility has been shown to be the preferred end point of VT ablation as it is associated with improved arrhythmia-free survival and all-cause mortality [4]. Therefore, even when performing substrate modification alone, one might still consider the need to induce VT in order to confirm abolition of all VTs. ECMO can prevent hemodynamic deterioration occurring during programmed stimulation.

The general increase in the prevalence of rapid and hemodynamically non-tolerated VTs, may result from the implementation of early coronary interventions for acute myocardial infarction in recent years [2]. Importantly, VT ablations are complex and prolonged procedures, typically lasting for several hours. Thus, many operators prefer to perform these procedures under general anesthesia (GA). However, many induced VTs under GA transpire as hemodynamically unstable [5]. This may limit the duration of the procedure, the feasibility of diagnostic maneuvers and the ability to reach the desired end point of non-inducibility. Thus, left ventricular hemodynamic support may be required in selected patients [6].

While often intravenous vasopressors may help 5, some high-risk patients might benefit from mechanical support. Several previous studies have shown that implementation of a peripheral percutaneous left ventricular assist devices (pLAVDs) allows more patients to undergo entrainment/ activation mapping, receive less rescue shocks and result in 
termination by ablation of more VTs during the procedure $[7,8]$. Notably, when comparing procedures performed with and without support there is yet no proof of improved outcome. However, most studies were underpowered to show such results. Furthermore, the retrospective nature of the studies precludes a robust comparison as patients treated with pLVAD may have been sicker [9]. Nevertheless, these results did suggest that the procedures supported by pLVAD are better tolerated, a fact that may be critical in patients with low cardiac reserve $[7,8]$. As pLVAD systems such as Impella of Tandem heart are not available in all countries, extracorporeal membrane oxygenation (ECMO) may be the only option for mechanical support in this setting. ECMO may provide the required hemodynamic support need for completion of this complex ablation in selected high risk patients. We present our initial experience using pre-procedure elective implementation ECMO assisted RFCA of scar related VT.

\section{Methods}

This study included patients that underwent VT ablation with ECMO support at our institute. All patients were indicated for the ablation procedure per current ESC guidelines $[10,11]$. Indications for ECMO assisted procedure were electrical storm, refractory to medical therapy that resulted in heart failure decompensation or, in one case hemodynamic compromise (premature ventricular contraction induced VF). All procedures were performed under GA with invasive arterial blood pressure monitoring.

Prior to the ablation procedure the left sided groin femoral artery and vein were surgically exposed. A short arterial cannula was inserted via the femoral artery (16/18 FR) and located in the external iliac artery. A long vein cannula (22/25 FR) was inserted via the femoral vein and located in the right atrium, under TEE guidance (Figure 1). Both cannulas were inserted with the Seldinger technique. The ECMO blood flow was maintained at a minimum of $1.5 \mathrm{~L} / \mathrm{min}$. In case of VT or $V F$ the blood flow on the ECMO machine was increased to 3 L/min to allow hemodynamic stability and adequate systemic organ perfusion.

\section{Electroanatomical mapping and quantitative assessment of low-voltage area}

The RF catheter was inserted into the LV by a retrograde aortic approach in one case (Patient 1 and 4) and a transseptal approach in the other two (Patients 2, 3 and 5). Epicardial mapping and ablation was carried out in one case (Patient 5). Electroanatomical mapping was performed (Carto 3 or XP, Biosense Webster, Diamond Bar, CA, USA) using a 3.5 $\mathrm{mm}$ tip open irrigated catheter (NaviStar ThermoCool, Biosense Webster, Diamond Bar Ca). Voltage maps were created during sinus rhythm. Peak-to-peak bipolar electrogram amplitude $<0.5 \mathrm{mV}$ was defined as dense scar, voltage $\geq 0.5$ and $<1.5 \mathrm{mV}$ as scar border zone [12].

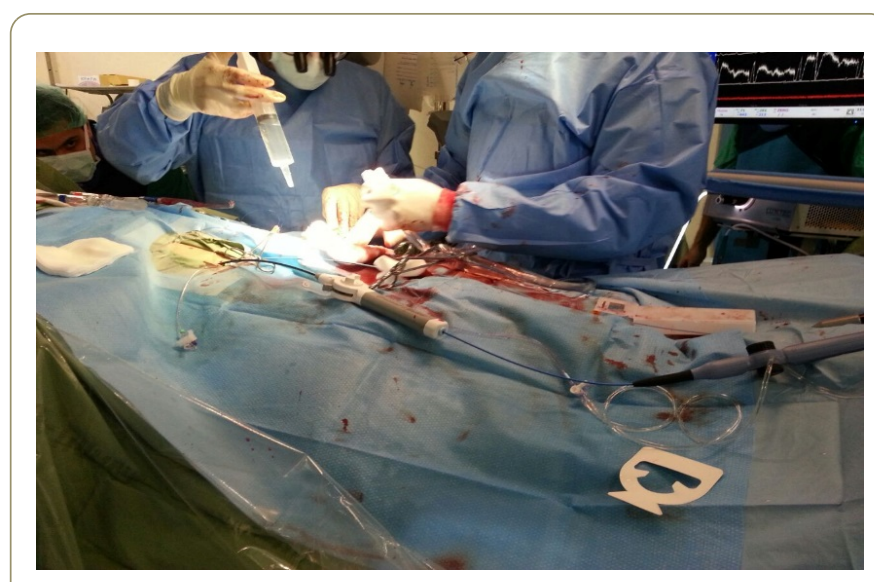

Figure 1 Picture of ablation catheter in deflectable sheath in right groin. The ECMO cannula are inserted in the left groin.

All inducible sustained monomorphic ventricular tachycardia (SMVT) were targeted for ablation. Sites were targeted for ablation if pacing entrained the SMVT with concealed fusion and a post-pacing interval (PPI) within $30 \mathrm{~ms}$ of the VT cycle length (CL) or by activation mapping. In any case scar substrate modification ("scar dechanneling") was performed. Sites with low amplitude fractionated electrograms that had long stimulation to QRS, late potentials or the best pace map sites were targeted. In the case of focal PVC ablation, the site of earliest pre-systolic electrical activity was targeted for ablation. Pace mapping and entrainment mapping utilized unipolar stimuli with strength of $10 \mathrm{~mA}$ and pulse width of $2 \mathrm{~ms}$ [10]. Radiofrequency (RF) energy was delivered at a power of 25 to 50 Watts targeting an impedance drop of at least 10 ohms. The endpoint of all procedures was non inducibility of any VT with programmed stimulation (PS) at a basic drive train of 600 $\mathrm{m} \mathrm{sec}$ and $400 \mathrm{~m} \mathrm{sec}$ with up to 3 extra stimuli. A procedure was defined as successful if the patients were non-inducible for any VT following PS. Partial successes were declared when the clinical VT was no longer inducible and acute procedural failure was declared if the clinical VT was still inducible.

Data regarding patients, demographics, clinical and procedural features were obtained from our VT ablation registry. All patients were followed by our cardiac implantable device clinic with repeated visits to the clinic which included clinical evaluation and device interrogations at 1 month, 3 months and every 6 months thereafter. The Institutional Review Board of Sheba Medical Center approved this registry on the basis of strict maintenance of participants' anonymity during database analyses. No individual consent was obtained.

A total of 8 VTs were targeted in 4 patients (median of 2 per patient). In another patient, we targeted PVC triggering recurrent VF. In all patients, extensive scar de-channeling was also performed. We were able to induce or document (in PVC case) the clinical ventricular arrhythmia. Mapping during VT was achieved in 4 VTs (3 clinical and one non-clinical VT). During VT, circulatory support up was titrated to a maximum of $4 \mathrm{~L} / \mathrm{min}$ to allow mapping and entrainment. 


\section{Results}

Patient's baseline clinical characteristics are described in

Table 1.

Table 1 Baseline clinical characteristics.

\begin{tabular}{|c|c|c|c|c|c|}
\hline & Patient 1 & Patient 2 & Patient 3 & Patient 4 & Patient 5 \\
\hline Age & 54 & 67 & 46 & 63 & 62 \\
\hline Sex & Male & Male & Male & Male & Male \\
\hline Cardiomyopathy & Ischemic & $\begin{array}{l}\text { Combined ischemic and } \\
\text { valvular }\end{array}$ & Ischemic & Ischemic & Idiopathic DCM \\
\hline Diabetes mellitus & $\sqrt{ }$ & - & - & - & - \\
\hline Hypertension & $\sqrt{ }$ & $\sqrt{ }$ & - & $\sqrt{ }$ & - \\
\hline Atrial fibrillation & - & $\sqrt{ }$ & - & $\sqrt{ }$ & - \\
\hline Hyperlipidemia & $\sqrt{ }$ & $\sqrt{ }$ & $\sqrt{ }$ & $\sqrt{ }$ & - \\
\hline NYHA functional class & III & II & II & III & III \\
\hline LVEF, \% & - & 25 & 20 & 15 & 15 \\
\hline Anticoagulation & - & warfarin & - & Warfarin & - \\
\hline $\begin{array}{l}\text { Pre-procedure Antiarrhythmic } \\
\text { therapy }\end{array}$ & $\begin{array}{l}\text { Amiodarone } \\
\text { +Procainamide }\end{array}$ & Amiodarone+lidocaine & $\begin{array}{l}\text { Amiodarone } \\
\text { +lidocaine }\end{array}$ & $\begin{array}{l}\text { Amiodarone } \\
\text { +lidocaine }\end{array}$ & Amiodarone+Mexilitin \\
\hline Pre-procedure-vasopressors & - & - & - & $\sqrt{ }$ & - \\
\hline
\end{tabular}

In those, the VT isthmus was mapped and found. RF at the critical VT isthmus site terminated the VT. Examples of an electronatomical map and pacemap are shown in Figure 2. In 4 other VTs (one clinical and 3 non-clinical VTs) we were not able to map during VT. In one case activation mapping was not performed since the induced VT was not sufficiently sustained to allow mapping and in 3 other cases mapping was not enabled due to very low local potentials and electrically unexcitable tissue that precluded any measurement or maneuvers. Therefore, we could not define the VT isthmus. Further substrate scar de-channeling enabled reaching the endpoint of non-inducibility for any VT in 4 patients. In a single case, one VT, albeit significantly faster than the clinical VT, was still inducible. In that particular case, ECMO support was increased to $4 \mathrm{~L} / \mathrm{min}$ to allow further substrate mapping but no additional relevant locations were found therefore, the procedure was concluded. Thus, it seemed that the clinical VT was ablated but a non-clinical morphology was still inducible. There were no cases of acute procedural failure.

\section{In-hospital course}

Four patients were successfully weaned from the ECMO circuit upon termination of the procedure with no need for inotropic support. With the exception of patient 4 , extubation was performed on the procedure table. No peri-procedural or femoral vascular complications occurred. These patients were discharged 4 to 13 days after the procedure with no vascular complications or additional VT. Patient 4 stayed on ECMO support for another 24 hours due to the combination of severe cardiogenic failure combined with hepatic and kidney failure (which was present upon admission). The ECMO was taken out 24 hours later but the patient needed both vassopressors and inotropes. His hepatic failure and cardiac output started to recover 48 hours after the procedure while remaining in normal sinus rhythm with no VT but then developed sepsis and unfortunately did not recover.

\section{Follow up}

All the other 4 patients were followed in our clinic including regular device interrogations. The median follow up was 16 months ( 1 to 23 months) after the index procedure (Table 3 ). All patients remained free from VT throughout follow up. Patients 1 and 2 remained ambulatory and required no repeated re-hospitalization to date. Patient 3 was admitted 4 months after the procedure and underwent implantation of a left ventricular assist device due to progressive heart failure but without VT. 


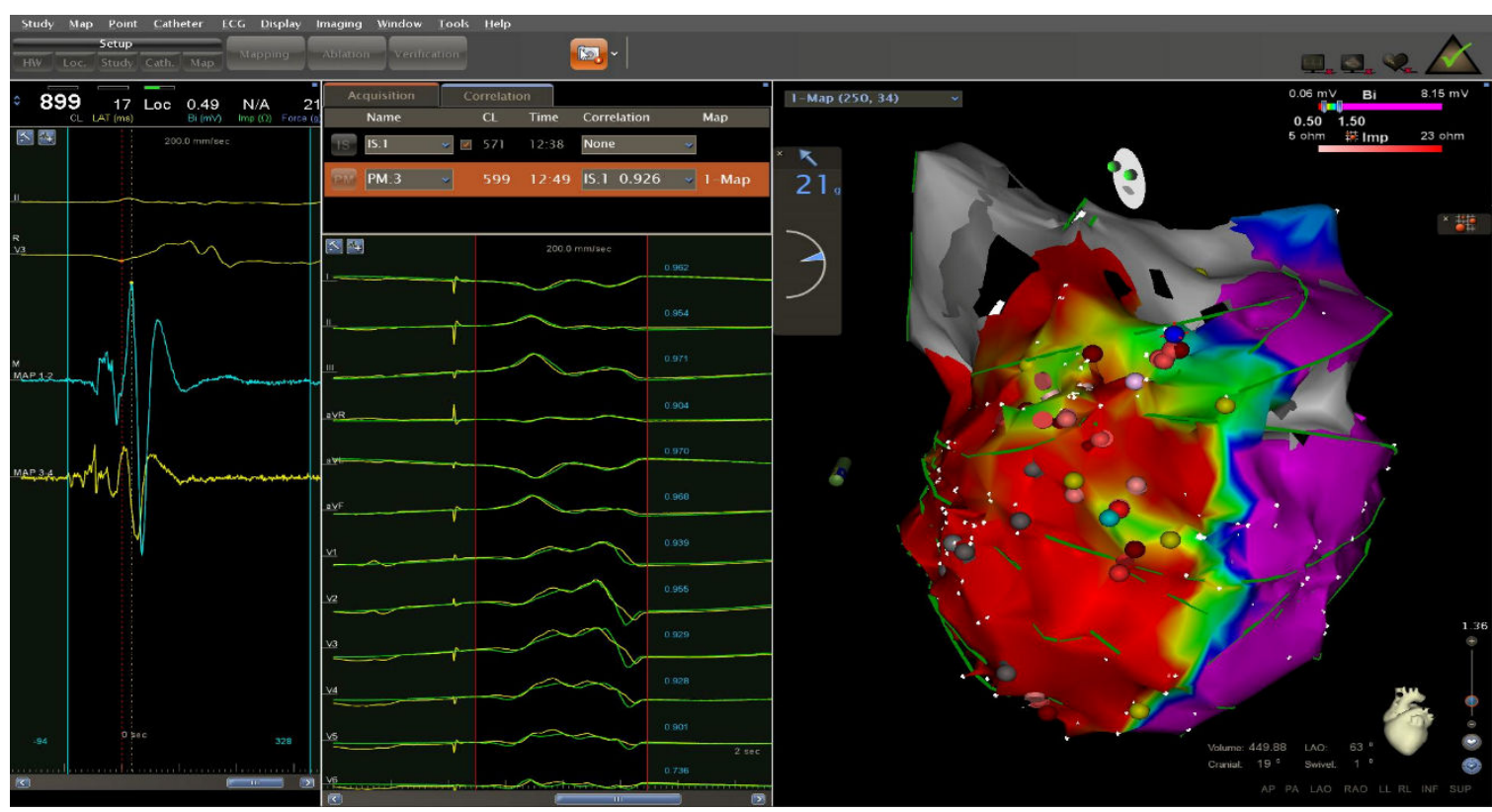

Figure 2 Electroanatomical map of patient number 3 demonstrating a large anterior wall low voltage area (in red). On the right side of the screen a good comparison of pacemap with clinical VT done with PASSO software can be seen.

The main findings and procedural information are described

in Table 2.

Table 2 Procedure related data.

\begin{tabular}{|c|c|c|c|c|c|}
\hline & Patient 1 & Patient 2 & Patient 3 & Patient 4 & Patient 5 \\
\hline $\begin{array}{l}\text { Index hospital stay } \\
\text { duration }\end{array}$ & 13 & 9 & 4 & 5 & 20 \\
\hline VT ablation approach & Retrograde & Trans-septal & Trans-septal & Retrograde & $\begin{array}{l}\text { Trans-septal and } \\
\text { epicardial }\end{array}$ \\
\hline $\begin{array}{l}\text { Main clinical VT/PVC } \\
\text { morphology }\end{array}$ & LB, IA & $\mathrm{RB}, \mathrm{EAD}$ & $\mathrm{LB}, \mathrm{SA}$ & $\mathrm{RB}, \mathrm{NA}$ & RB, EAD \\
\hline Scar location & Apicoseptal & Posteriobasal & Antero-septal & Septoapical & none \\
\hline $\begin{array}{l}\text { Number of VT/PVC } \\
\text { morphologies }\end{array}$ & 1 & 1 & 1 & 3 & 3 \\
\hline Clinical VT/ PVC induced & $\sqrt{ }$ & $\sqrt{ }$ & $\sqrt{ }$ & $\sqrt{ }$ & $\sqrt{ }$ \\
\hline $\begin{array}{l}\text { Number of non- Clinical } \\
\text { VTs observed induced }\end{array}$ & 0 & 0 & 0 & 2 & 2 \\
\hline Critical isthmus located & NA & 1 & 0 & 2 & 3 \\
\hline $\begin{array}{l}\text { No. of VT termination by } \\
\text { isthmus ablation }\end{array}$ & NA & 1 & 0 & 1 & 2 \\
\hline $\begin{array}{l}\text { No. of VT targeted with } \\
\text { substrate modification }\end{array}$ & NA & 0 & 1 & 2 & 1 \\
\hline $\begin{array}{l}\text { Procedure duration } \\
\text { (minute) }\end{array}$ & 445 & 353 & 335 & 270 & 435 \\
\hline $\begin{array}{l}\text { Acute } \\
\text { outcome }\end{array}$ & Complete Success & Partial success & Complete Success & Complete Success & Complete Success \\
\hline
\end{tabular}




\section{Discussion}

We present our initial experience with patients undergoing ECMO insertion in order to allow assisted RFCA of patients during refractory electrical storm. In all cases the use of ECMO was planned and implemented specifically to support the procedure itself and not beforehand or as a bail-out strategy for dealing with sudden hemodynamic compromise arising during the procedure. All 5 cases presented complex clinical challenges with very limited therapeutical options and high risk for mortality.

Table 3 Procedure related data.

\begin{tabular}{|c|c|c|c|c|c|}
\hline & Patient 1 & Patient 2 & Patient 3 & Patient 4 & Patient 5 \\
\hline Index hospital stay duration & 13 & 9 & 4 & 5 & 20 \\
\hline VT ablation approach & Retrograde & Trans-septal & Trans-septal & Retrograde & $\begin{array}{l}\text { Trans-septal and } \\
\text { epicardial }\end{array}$ \\
\hline $\begin{array}{l}\text { Main clinical VT/PVC } \\
\text { morphology }\end{array}$ & LB, IA & $\mathrm{RB}, \mathrm{EAD}$ & $\mathrm{LB}, \mathrm{SA}$ & $\mathrm{RB}, \mathrm{NA}$ & $\mathrm{RB}, \mathrm{EAD}$ \\
\hline Scar location & Apicoseptal & Posteriobasal & Anteroseptal & Septoapical & none \\
\hline $\begin{array}{l}\text { Number of } \quad \text { VT/PVC } \\
\text { morphologies }\end{array}$ & 1 & 1 & 1 & 3 & 3 \\
\hline Clinical VT/ PVC induced & $\sqrt{ }$ & $\sqrt{ }$ & $\sqrt{ }$ & $\sqrt{ }$ & $\sqrt{ }$ \\
\hline $\begin{array}{l}\text { Number of non- Clinical VTs } \\
\text { observed induced }\end{array}$ & 0 & 0 & 0 & 2 & 2 \\
\hline Critical isthmus located & NA & 1 & 0 & 2 & 3 \\
\hline $\begin{array}{l}\text { No. of VT termination by } \\
\text { isthmus ablation }\end{array}$ & NA & 1 & 0 & 1 & 2 \\
\hline $\begin{array}{l}\text { No. of VT targeted with } \\
\text { substrate modification }\end{array}$ & NA & 0 & 1 & 2 & 1 \\
\hline Procedure duration (minute) & 445 & 353 & 335 & 270 & 435 \\
\hline Acute procedural outcome & Complete Success & Partial success & Complete Success & Complete Success & Complete Success \\
\hline
\end{tabular}

With the increased hemodynamic support, we could carry out sufficient mapping allowing the ablation of all inducible VTs. A total of 8 VTs were targeted in 4 patients. In most VTs the probable critical isthmus was found with combination of activation mapping even if limited or suggested by entrainment. RFCA at site of isthmus terminated VT in all cases. In patient number 3 , once induced, the clinical VT never persisted long enough to allow complete mapping but ECMO enabled to perform a detailed substrate mapping and provided support during short VT events. Finally, the ECMO allowed us to carry out a complete PS protocol up to a basic drive of 350 $\mathrm{m}$ sec with 4 extra stimuli in a patient who would not have tolerated such a PS otherwise due to overt heart failure. In our experience the use of ECMO for the duration of the procedure prompted lengthy procedures, enabled extensive mapping, identification of multiple VT isthmuses and aggressive PS thus promoting the desired endpoint of non-inducibility in a single procedure.

The risk of hemodynamic compromise during scar related VT ablation requiring mechanical support or procedure discontinuation has been recently evaluated by Santangeli et al. They reported a predictable increased risk of mortality it those patients [6]. Despite this, Santangeli, et al. reported $70 \%$ of VT non-inducibility at PS6. Notably their report described the use of Impella support and not ECMO.

Data describing the use of mechanical circulatory support for scar related VT ablation is scarce. Patients undergoing scar related VT ablation are per definition very sick. The risk of inducing VT leading to hemodynamic compromise is significant in any VT ablation procedure regardless of the techniques used.

Therefore, some advocate the use of mechanical hemodynamic support for every VT ablation procedure [8]. However, in a single study this was not proven beneficial [9]. Yet, it seems that mechanical support may be needed in a select group of patients. In our opinion, this includes patients with severe decompensated heart failure, or those with unstable VTs after failed substrate approach as well as patients with severe pulmonary hypertension or aortic stenosis who might not tolerate VT $[13,14]$.

The concept of using mechanical support for VT ablation has been previously reported $[8,14]$, describing the utilization of the Impella 2.5 systems in this setting. This device was found to be superior to vassopressors alone but inferior to peripheral cardio pulmonary by pass ( $\mathrm{CPB}$ ) specifically in a group of 
advanced heart failure and unstable VT storm [15]. The Impella device, currently unavailable in Israel and accordingly could not be used by us. However, it seems reasonable that many of those patients would benefit from full circulatory support in order to withstand the strain of RFCA and achieving the goal of non-inducibility. An additional advantage is that unlike the Impella, ECMO does not require hardware insertion into the LV chamber which potentially could limit catheter manipulation and have a pro arrhythmic effect.

While there are, several reports describing successful VT, ablations performed in hemodynamically unstable patients in electrical storm already supported by ECMO [16-20], to the extent of our knowledge, very few described the utilization of an ECMO circuit specifically to facilitate the ablation procedure [21]. The most comprehensive work in this field was published by Della Bella and colleagues describing the use of ECMO in the VT ablation of 28 high risk patients with various degrees of aortic insufficiency [22]. This report focused on hemodynamic effect of aortic insufficiency on the ECMO system. Regardless, ECMO was found to provide adequate circulatory support for the procedures.

Our report is in line with the above and demonstrated the advantage of ECMO in allowing detailed and lengthy procedures which would not have been otherwise possible. We also report on the use of ECMO for PVC/ RFCA induced VF. None of the patients had an indication for ECMO support prior to the procedure and ECMO was implanted only for the procedure. Four patients were weaned of ECMO on the procedure table and one 24 hrs afterwards.

The use of ECMO, however, has a few significant drawbacks and potential risks not the least of which are vascular access site complications and significant bleeding. Other potential complications include lower extremity ischemia, compartment syndrome, acute kidney injury and significant infections $[23,24]$. The procedure is time consuming and requires additional staff members with the relevant expertise. All of these, together with prolonged hospital stay, translate into significant costs. These are offset by the ECMOs ability to allow the maintenance of hemodynamic stability and end-organ perfusion during ongoing VT thereby facilitating activation and entrainment mapping. The LV unloading during periods of sinus rhythm to minimizes peri- and post-procedure worsening of heart failure.

Therefore, we suggest considering the use of peripheral left ventricular assist devices support for scar related VT ablation in selected patients which are unlikely to sustain such procedures without support. Risk stratification and early identification of these patients is key and consideration should be given to systemic medical conditions, structural heart disease, electrophysiological features and procedural-specific variables. It is equally important to identify patients in which a successful VT ablation may not alter the clinical course and prevent deterioration and mortality. In this small series although the ECMO allowed total performance of a full procedure, one patient died during the index hospitalization. Perhaps proving that for some VT is a sign of heart failure ("burned out heart") and eliminating the VT does always solve the problem.

\section{Conclusions}

We report our first experience of VT RFCA with ECMO support in treating patients with hemodynamically unstable electrical storm. ECMO support has allowed full VT mapping and ablation. Its implementation is safe and prevents hemodynamic collapse during the procedure. A strong cooperation between electrophysiologists and cardiac surgery is pivotal to achieve satisfactory results.

\section{References}

1. Pedersen CT, Kay GN, Kalman J, Borggrefe M, Della-Bella P, et al. (2014) EHRA/HRS/APHRS expert consensus on ventricular arrhythmias. Heart rhythm. J Heart Rhythm Soc 11: e166-196.

2. Josephson ME, Anter E (2015) Substrate mapping for ventricular tachycardia assumptions and misconceptions. Clin Electrophysiol 1: 341-352.

3. Santangeli P, Marchlinski FE (2015) Substrate mapping for unstable ventricular tachycardia. Heart rhythm.

4. Ghanbari H, Baser K, Yokokawa M, Stevenson W, Della Bella P, et al. (2014) Noninducibility in postinfarction ventricular tachycardia as an end point for ventricular tachycardia ablation and its effects on outcomes: a meta-analysis. Circ Arrhythm Electrophysiol 7: 677-683.

5. Nof E, Reichlin T, Enriquez AD, Ng J, Nagashima K, et al. (2015) Impact of general anesthesia on initiation and stability of VT during catheter ablation. Heart rhythm 12: 2213-2220.

6. Santangeli P, Muser D, Zado ES, Magnani S, Khetpal S, et al. (2015) Acute hemodynamic decompensation during catheter ablation of scar-related ventricular tachycardia: incidence, predictors, and impact on mortality. Circ Arrhythm Electrophysiol 8: 68-75.

7. Reddy YM, Chinitz L, Mansour M, Bunch TJ, Mahapatra S, et al. (2014) Percutaneous left ventricular assist devices in ventricular tachycardia ablation: multicenter experience. Circ Arrhythm Electrophysiol 7: 244-250.

8. Miller MA, Dukkipati SR, Mittnacht AJ, Chinitz JS, Belliveau L, et al. (2011) Activation and entrainment mapping of hemodynamically unstable ventricular tachycardia using a percutaneous left ventricular assist device. J Am Coll Cardiol 58:1363-1371.

9. Bunch TJ, Darby A, May HT, Ragosta M, Lim DS, et al. (2012) Efficacy and safety of ventricular tachycardia ablation with mechanical circulatory support compared with substrate-based ablation techniques. Europace 14: 709-714.

10. Aliot EM, Stevenson WG, Almendral-Garrote JM, Bogun F, Calkins $\mathrm{CH}$, et al. (2009) HRA/HRS expert consensus on catheter ablation of ventricular arrhythmias: Developed in a partnership with the European heart rhythm association (EHRA), a registered branch of the European society of cardiology (ESC), and the heart rhythm society (HRS); in collaboration with the American college of cardiology (ACC) and the American heart association (AHA). Heart rhythm 6: 886-933.

11. Priori SG, Blomström-Lundqvist C, Mazzanti A, Blom N, Borggrefe M, et al. (2015) ESC Guidelines for the management 
of patients with ventricular arrhythmias and the prevention of sudden cardiac death: The task force for the management of patients with ventricular arrhythmias and the prevention of sudden cardiac death of the European society of cardiology (ESC)Endorsed by: Association for European paediatric and congenital cardiology (AEPC). Eur Heart J 36: 2793-2867

12. Marchlinski FE, Callans DJ, Gottlieb CD, Zado E (2000) Linear ablation lesions for control of unmappable ventricular tachycardia in patients with ischemic and nonischemic cardiomyopathy. Circulation 101: 1288-1296.

13. Katrina Mountfort MWRC, Kirchhof P (2013) The European heart rhythm association practical guide on the use of new oral anticoagulants in patients with non-valvular atrial fibrillation: A brief summary. Arrhythm Electrophysiol Rev 2: 115-119.

14. Abuissa H, Roshan J, Lim B, Asirvatham SJ (2010) Use of the Impella microaxial blood pump for ablation of hemodynamically unstable ventricular tachycardia. J Cardiovasc Electrophysiol 21: 458-461.

15. Lu F, Eckman PM, Liao KK, Apostolidou I, John R, et al. (2013) Catheter ablation of emodynamically unstable ventricular tachycardia with mechanical circulatory support. Int J Cardiol 168: 3859-3865.

16. Couto-Mallon D, Ariza-Sole A, Guerrero C, Muntane G, Dallaglio $P D$, et al. (2016) Extracorporeal membrane oxygenation ventricular support as a bridge to ablation in refractory cardiogenic shock refractory to tachycardia-induce cardiomyopathy. Med Intensiva 40: 62-64.

17. Rizkallah J, Shen S, Tischenko A, Zieroth S, Freed DH, et al. (2013) Successful ablation of idiopathic left ventricular tachycardia in an adult patient during extracorporeal membrane oxygenation treatment. Can J Cardiol 29: 1741 e1717-1749.
18. Ucer E, Fredersdorf S, Jungbauer C, Debl K, Philipp A, et al. (2014) A unique access for the ablation catheter to treat electrical storm in a patient with extracorporeal life support. Europace 16:299-302.

19. Scherrer V, Lasgi C, Hariri S, Dureuil B, Savoure A, et al. (2012) Radiofrequency ablation under extracorporeal membrane oxygenation for atrial tachycardia in postpartum. J Card Surg 27: 647-649.

20. Della Bella P, Baratto F, Tsiachris D, Trevisi N, Vergara P, et al. (2013) Management of ventricular tachycardia in the setting of a dedicated unit for the treatment of complex ventricular arrhythmias: long-term outcome after ablation. Circulation 127: 1359-1368.

21. Cheruvu C, Walker B, Kuchar D, Subbiah RN (2014) Successful ablation of incessant $\mathrm{AV}$ reentrant tachycardia in a patient on extracorporeal membrane oxygenation. Heart Lung Circ 23: e12-15.

22. Pappalardo F, Regazzoli D, Mangieri A, Ajello S, Melisurgo G, et al. (2016) Hemodynamic and echocardiographic effects of aortic regurgitation on femoro-femoral veno-arterial ECMO. Int J Cardiol 202: 760-762.

23. Hummel JD, Strickberger SA, Daoud E, Niebauer M, Bakr O, et al. (1994) Results and efficiency of programmed ventricular stimulation with four extrastimuli compared with one, two, and three extra stimuli. Circulation 90: 2827-2832.

24. Cheng R, Hachamovitch R, Kittleson M, Patel J, Arabia F, et al. (2014) Complications of extracorporeal membrane oxygenation for treatment of cardiogenic shock and cardiac arrest: a metaanalysis of 1,866 adult patients. Ann Thorac Surg 97: 610-616. 